\title{
Verifying the Accuracy of Digital Goniometer Range of Motion Measurements for In-Person and Telemedicine Visits
}

\author{
Zack Langton $^{1}$, Mari Johnson ${ }^{1 *}$, Zach Reed ${ }^{1}$, John Alchemy ${ }^{2 *}$, Jerry Artz $^{1}$ and Bruce Bolon ${ }^{1}$ \\ ${ }^{1}$ Department of Physics, Hamline University, Saint Paul, Minnesota, USA \\ ${ }^{2}$ RateFast Corporation, Santa Rosa, USA
}

Correspondence should be addressed to John Alchemy, j.alchemy@pr4report.com

Received: August 25, 2020; Accepted: September 14, 2020; Published: September 21, 2020

\begin{abstract}
$\underline{\text { ABSTRACT }}$
INTRODUCTION

This paper offers empirical evidence of the accuracy of the clinical application of the RateFast Goniometer smartphone app. Using multiple comparative measures and interrater reliability measures, this paper investigates the effectiveness of this digital goniometer app for physicians practicing in both the office and in telemedicine clinical settings.
\end{abstract}

\section{METHODS}

Three experiments were performed to test the reliability of the RateFast Goniometer app. The first involved measuring preset angles to test its accuracy. The second experiment involved measuring randomly drawn angles to determine if switching users has any effect on the results. The last experiment measured shoulder angles (flexion and extension planes) of 53 volunteers to determine the accuracy of the RateFast Goniometer app in both haptic mode (for use in an in-person clinical setting) and camera mode (for use in a telemedicine clinical setting).

\section{RESULT}

In the first experiment, the average difference between measurements was $0.6^{\circ}$ and the average standard deviation was $0.3^{\circ}$. In the second experiment, the angles measured with the RateFast goniometer were less than those measured with a protractor, averaging to a difference of $0.9^{\circ}$. In the third experiment, the haptic mode measurements and the camera mode measurements had an average difference of $1.2^{\circ}$ and the standard deviation of the difference between haptic and camera measurements was determined to be $4.7^{\circ}$ across all volunteers.

\section{CONCLUSION}

In all three experiments, the error rate found using the RateFast Goniometer app which is within the error tolerance according to the AMA Guides to the Evaluation of Permanent Impairment, Fifth Edition (AMA Guides), which stipulates that measurements of shoulder angles must be within $10 \%$ of one another. The RateFast Goniometer app and similar digital goniometer applications can be used to accurately measure angles in both in-person and telemedicine settings according to the standards of accuracy set forth in the AMA Guides.

Citation: Zack Langton, Verifying the Accuracy of Digital Goniometer Range of Motion Measurements for In-Person and Telemedicine Visits. J Clin Cases Rep 3(S3): 31-39. 


\section{KEYWORDS}

Digital goniometer; Telemedicine; Occupational medicine; Orthopedic medicine

\section{INTRODUCTION}

A goniometer is a device used to measure angles and is therefore useful in quantifying range of motion in athletic training and health-related fields such as physical therapy, orthopedic medicine, and occupational medicine. Various descriptions regarding the correct use of traditional goniometers exist in the literature [1].

In recent years, smartphone apps have been developed to replace traditional goniometers. These goniometer applications use the smartphones' accelerometers to measure angles. One 2014 study [2] compared the measurements between a smartphone goniometer app and a traditional goniometer by measuring the range of motion of the knees of 36 volunteers performing three standing lunges. No significant difference was found between the measurements obtained using the smartphone goniometer and the universal goniometer.

Here, we take our standard for a "significant" difference between two measurements in clinical practice from the AMA Guides to the Evaluation of Permanent Impairment, Fifth Edition (hereafter referred to as the AMA Guides), which is a widely used standard in the United States for determining patients' impairment in workers' compensation and disability claims. When measuring the range of motion of patients' joints, the AMA Guides allows for a difference between two angle measurements of up to 10\%; therefore, any difference in two measurements greater than $10 \%$ is considered a "significant" difference in this context [3].

The smartphone application used in this study is the RateFast Goniometer app, which has two modes of operation [4]. One is the default haptic mode, useful for measuring angles in-person, as was done in the 2014 study mentioned above. The other mode is the camera mode, which can be used for measuring angles for analysis by a remote diagnostician, such as in telemedicine [5].

To date, little evidence exists in the literature that confirms the accuracy of data obtained using a goniometer in a telemedicine setting. One study describes the accuracy of a traditional goniometer [6]. Another publication verified the use of machine learning software for measuring the range of motion of a patient's shoulder in telemedicine [7]. A third publication, in which elbow flexion and extension was measured, found that telemedicine-based goniometry is possible [8]. Although there has been little work done involving range of motion measurements in telemedicine, many studies demonstrate the validity of telemedicine in general. One study examining 200 patients for various issues compared the results from face-to-face consultations to telemedicine consultations and found no significant difference [9]. Several other publications discuss the efficacy of telemedicine in greater detail [10-26].

For many disability and workers' compensation claims, range of motion measurements are a critical factor to determine the level of a patient's impairment. As many patients do not have easy access to a physician's officesuch as patients living in rural areas, or patients living far away from an in-network physician who accepts workers' compensation cases-a physician's ability to measure a patient's range of motion from a remote location is a valuable tool in clinical practice. To test the accuracy of measuring a patient's range of motion remotely, a concomitant study used the RateFast goniometer app in both haptic and camera modes to determine range of motion for flexion and extension for healthy individuals between the ages of 18 years - 24 years [5]. Data from 
these experiments are described in the results of the current study.

\section{$\underline{\text { METHODS }}$}

Three separate experiments were conducted to determine the accuracy of the measurements obtained using the RateFast goniometer app in both in-person and telemedicine clinical settings. Prior to taking measurements for this study, three student researchers underwent training in the use of the RateFast goniometer app by watching a RateFast educational video featuring a qualified medical evaluator demonstrating the correct operation of the RateFast goniometer app [4]. The smartphone models used in all three experiments were either the iPhone 8 Plus or the iPhone $\mathrm{X}$, each running version 1.3 of the RateFast goniometer app.
The first experiment tested the accuracy of the RateFast goniometer app when measuring verified angles on a flat surface. A fluid carpenter's level was used to draw a horizontal line on a whiteboard. Additional lines were drawn using a protractor creating angles of 0 degree, 30 degrees, 45 degrees, 60 degrees, 90 degrees, 120 degrees, 135 degrees, 150 degrees and 180 degrees. One student researcher then measured each angle twice using his or her own smartphone with the RateFast goniometer app. Then, to account for variance among users and devices, the student researcher proceeded to use the RateFast goniometer app with the other student researchers' smartphones to measure the same angles again. The remaining two student researchers did the same, with results shown in Table 1.

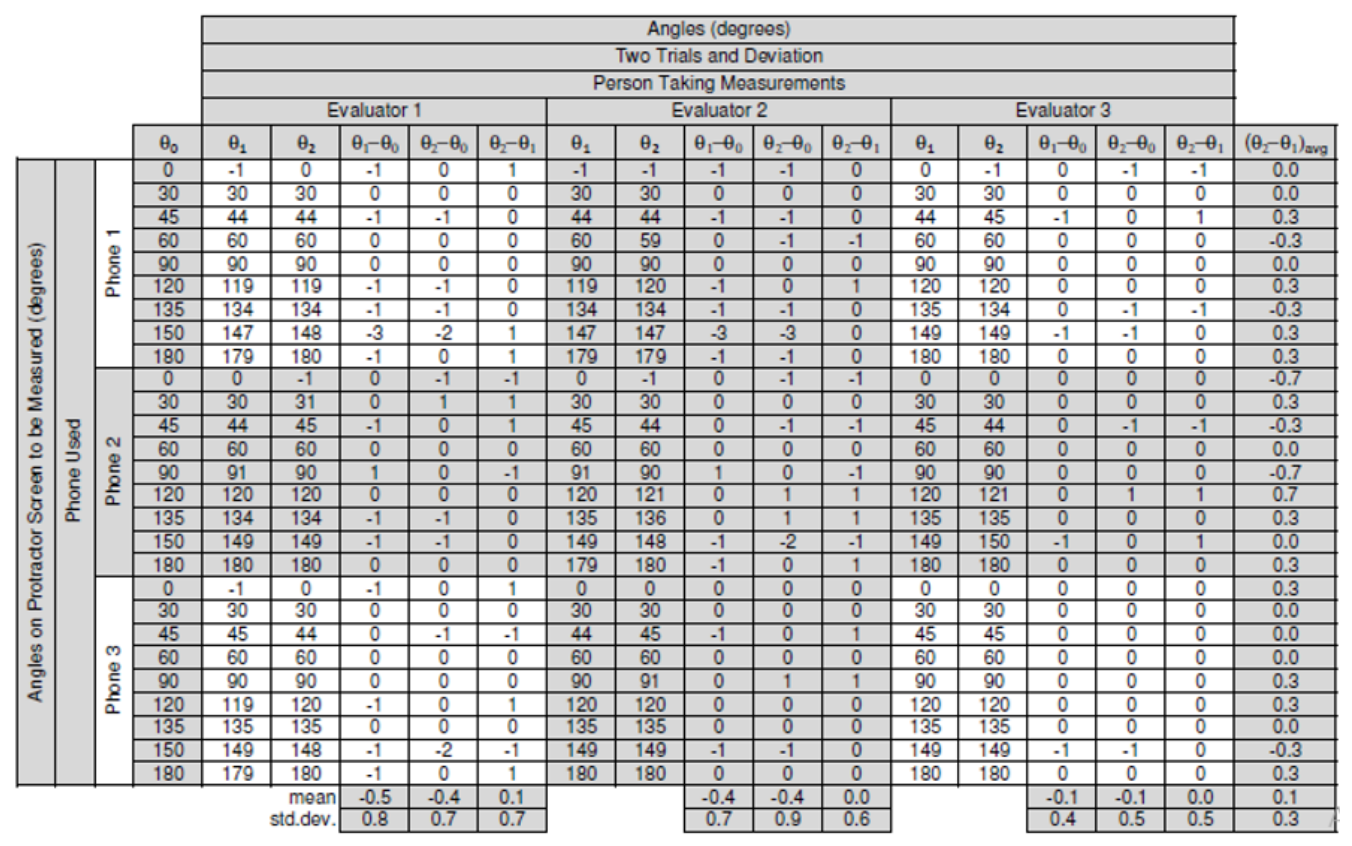

Table 1: Angle measurements using the RateFast app for angles drawn on a whiteboard with a protractor. Each student made two measurements $\left(\theta_{1} \& \theta_{2}\right)$ of each angle drawn $\left(\theta_{0}\right)$ using each of the three smartphones.

Experiment two was designed to expand upon the results obtained in the first experiment. One researcher drew random lines and measured the angle of each line using a protractor. The other two researchers separately used the RateFast goniometer app to measure the angles. To limit bias, each of the three measurements was made without the researcher knowing the results of the measurements taken with the other tool. The results obtained using the goniometer were then compared to the reference values obtained using the protractor, as shown in Table 2. 


\begin{tabular}{|c|c|c|c|c|c|}
\hline \multicolumn{6}{|c|}{ Angles (degrees) } \\
\hline$\theta_{0}$ & $\theta_{1}$ & $\theta_{2}$ & $\theta_{1}-\theta_{0}$ & $\theta_{2}-\theta_{0}$ & $\theta_{2}-\theta_{1}$ \\
\hline 10 & 9 & 9 & -1 & -1 & 0 \\
\hline 26 & 26 & 27 & 0 & 1 & 1 \\
\hline 50 & 47 & 50 & -3 & 0 & 3 \\
\hline 71 & 69 & 69 & -2 & -2 & 0 \\
\hline 99 & 98 & 98 & -1 & -1 & 0 \\
\hline 15 & 13 & 12 & -2 & -3 & -1 \\
\hline 29 & 24 & 27 & -5 & -2 & 3 \\
\hline 42 & 40 & 41 & -2 & -1 & 1 \\
\hline 78 & 77 & 76 & -1 & -2 & -1 \\
\hline 95 & 93 & 94 & -2 & -1 & 1 \\
\hline 25 & 23 & 25 & -2 & 0 & 2 \\
\hline 41 & 40 & 42 & -1 & 1 & 2 \\
\hline 56 & 55 & 57 & -1 & 1 & 2 \\
\hline 77 & 74 & 75 & -3 & -2 & 1 \\
\hline 110 & 108 & 109 & -2 & -1 & 1 \\
\hline 35 & 35 & 34 & 0 & -1 & -1 \\
\hline 63 & 62 & 62 & -1 & -1 & 0 \\
\hline 93 & 92 & 92 & -1 & -1 & 0 \\
\hline 115 & 112 & 112 & -3 & -3 & 0 \\
\hline 129 & 127 & 126 & -2 & -3 & -1 \\
\hline 22 & 22 & 21 & 0 & -1 & -1 \\
\hline 37 & 37 & 36 & 0 & -1 & -1 \\
\hline 56 & 56 & 56 & 0 & 0 & 0 \\
\hline 86 & 86 & 86 & 0 & 0 & 0 \\
\hline 102 & 100 & 102 & -2 & 0 & 2 \\
\hline 8 & 8 & 10 & 0 & 2 & 2 \\
\hline 40 & 38 & 39 & -2 & -1 & 1 \\
\hline 73 & 72 & 74 & -1 & 1 & 2 \\
\hline 106 & 110 & 108 & 4 & 2 & -2 \\
\hline 130 & 132 & 129 & 2 & -1 & -3 \\
\hline \multirow{2}{*}{\multicolumn{3}{|c|}{$\begin{array}{l}\text { mean } \\
\text { std.dev. }\end{array}$}} & -1.1 & -0.7 & 0.4 \\
\hline & & & 1.6 & 1.3 & 1.5 \\
\hline
\end{tabular}

Table 2: Angle measurements using the RateFast app for random angles drawn on a whiteboard. Each angle was measured with a protractor $\left(\theta_{0}\right)$ by one student researcher, then independently measured by two other student researchers (angles labeled $\theta_{1}$ and $\theta_{2}$ ).

The third and final experiment was designed to verify the accuracy of the RateFast goniometer app when measuring the range of motion of patients in both inperson and telemedicine clinical settings. 53 volunteers were gathered from the Hamline University community for the purpose of testing the accuracy of the Rate-Fast goniometer app and, in a separate experiment [5], performing shoulder range of motion studies with and without a TheraBand (an elastic band to measure strength-testing or for use in physical therapy). Although the TheraBand and other factors affecting shoulder range of motion are not the focus of this paper, the need for sufficient data to investigate such dependences and additional clinical variables would provide several hundred measurements that can be used to statistically verify the accuracy of the RateFast goniometer app.
In this third experiment, the RateFast goniometer app was used to measure the flexion and extension in the sagittal plane of each volunteer's shoulder, which involves measuring the angle formed by the volunteer's arm and the torso (the torso serves as the $0^{\circ}$ plane of reference). The arm was extended forward with the elbow fully extended and the thumb pointed upward. This is the method of flexion and extension measurement specified by the AMA Guides [3].

Before beginning the measurements, each volunteer was asked to do two sets of flexion and extension warm-up stretches. Data collection began with the volunteer reaching the terminal angle of the arm in both flexion and extension twice for each arm, first with and then without the TheraBand resistance device, for a total of 16 angle measurements. With the volunteer's arm at the terminal angle of flexion or extension, the angle was measured in haptic-mode by placing the smartphone running the RateFast goniometer app on the mid-bicep and the elbow while at the terminal flexion or extension (Figure 1 and Figure 2). An attempt was made to perform the experiment with the smartphone strapped to the arm, but it was observed that the goniometer rotation axis did not remain perpendicular to the sagittal plane both in flexion and extension (i.e., rotated away from horizontal). Therefore, the measurements were taken using the goniometer after the terminal angle was reached.

For the camera mode measurements, a picture was taken using a second smartphone on a tripod, and a third smartphone was used to obtain videos of the measurement-taking process. The second smartphone (used for pictures) was positioned at a distance of 12 feet from the volunteer, which safely allowed the camera to capture the entire volunteer with his or her arm fully extended, and at a height aligned with the volunteer's shoulder to minimize parallax issues. 


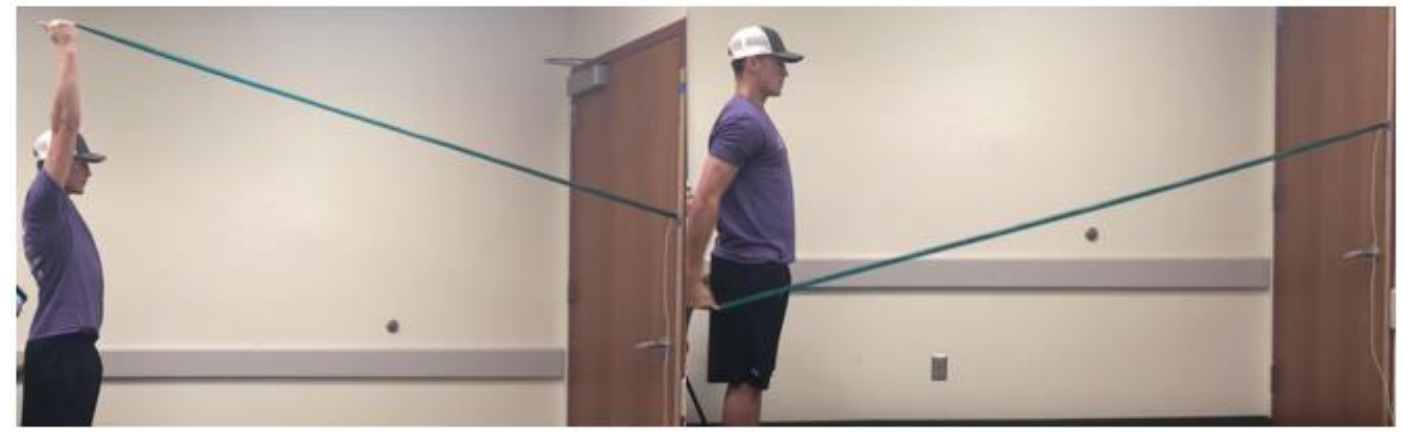

Figure 1: Flexion (left) and extension (right) in the sagittal plane.

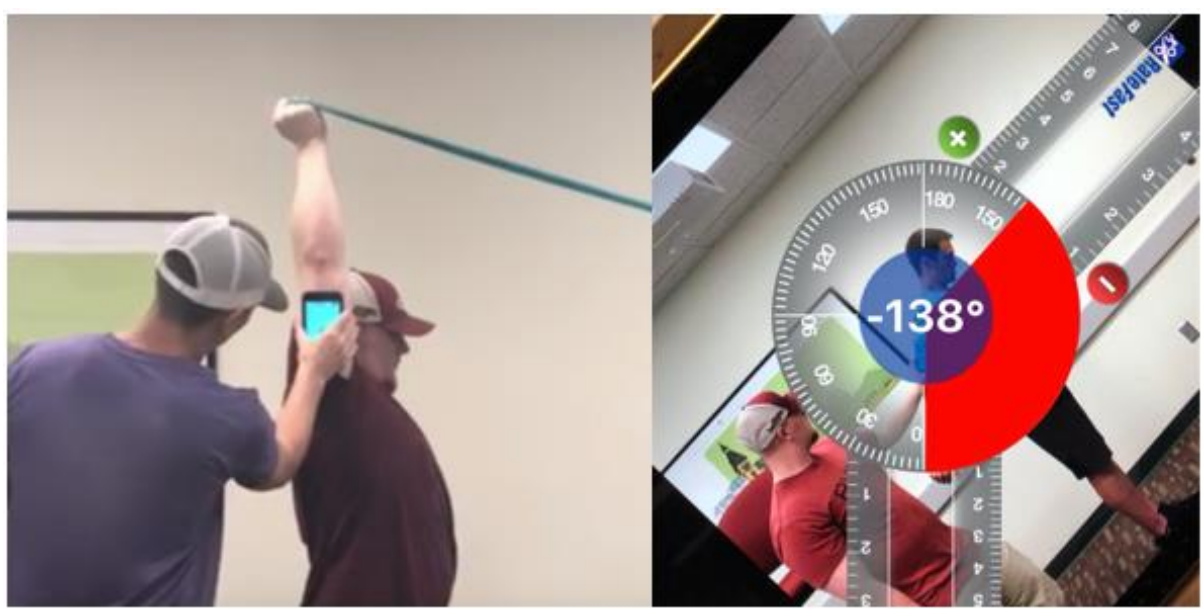

Figure 2: Using the RateFast goniometer app in (left) "haptic mode" to take in-person measurements, and (right) "camera mode" to take telemedicine measurements.

The camera was positioned at the volunteer's shoulder height because it was determined that parallax contributed insignificantly if the camera was positioned at a height no lower than the waist and no higher than the head. The test to determine the contribution of parallax on angle measurements was performed by altering the height of the camera while measuring the angle at which a student researcher held their arm. The arm of the student researcher was placed against a poster board with lines drawn at 10 degree increments above and below the horizon, thereby determining the angle at which the arm was held. The camera was placed 12 feet away from the subject's shoulder and initially set at the same height of the shoulder. The camera was then raised and lowered at 1-inch increments with a picture taken at each height. The angle of the arm in each picture was then measured using the RateFast goniometer app in camera mode. The
RateFast goniometer app camera mode measurements were then compared to the known angle of the arm. The difference between the known angle and the measurements obtained from the picture using the RateFast goniometer app only became significant once the camera height fell below the subject's waist or rose above their head.

Once all 16 measurements were taken, the angles were re-measured by analyzing the pictures on a computer screen using the camera function on the RateFast goniometer (Figure 2). This was done to measure the reliability of the measurements obtained using the camera mode of the RateFast goniometer app in a telemedicine scenario, such as a medical practitioner measuring the image of a patient on a computer screen. The result for this experiment can be seen in Table $3 a$ \& Table $3 b$. 

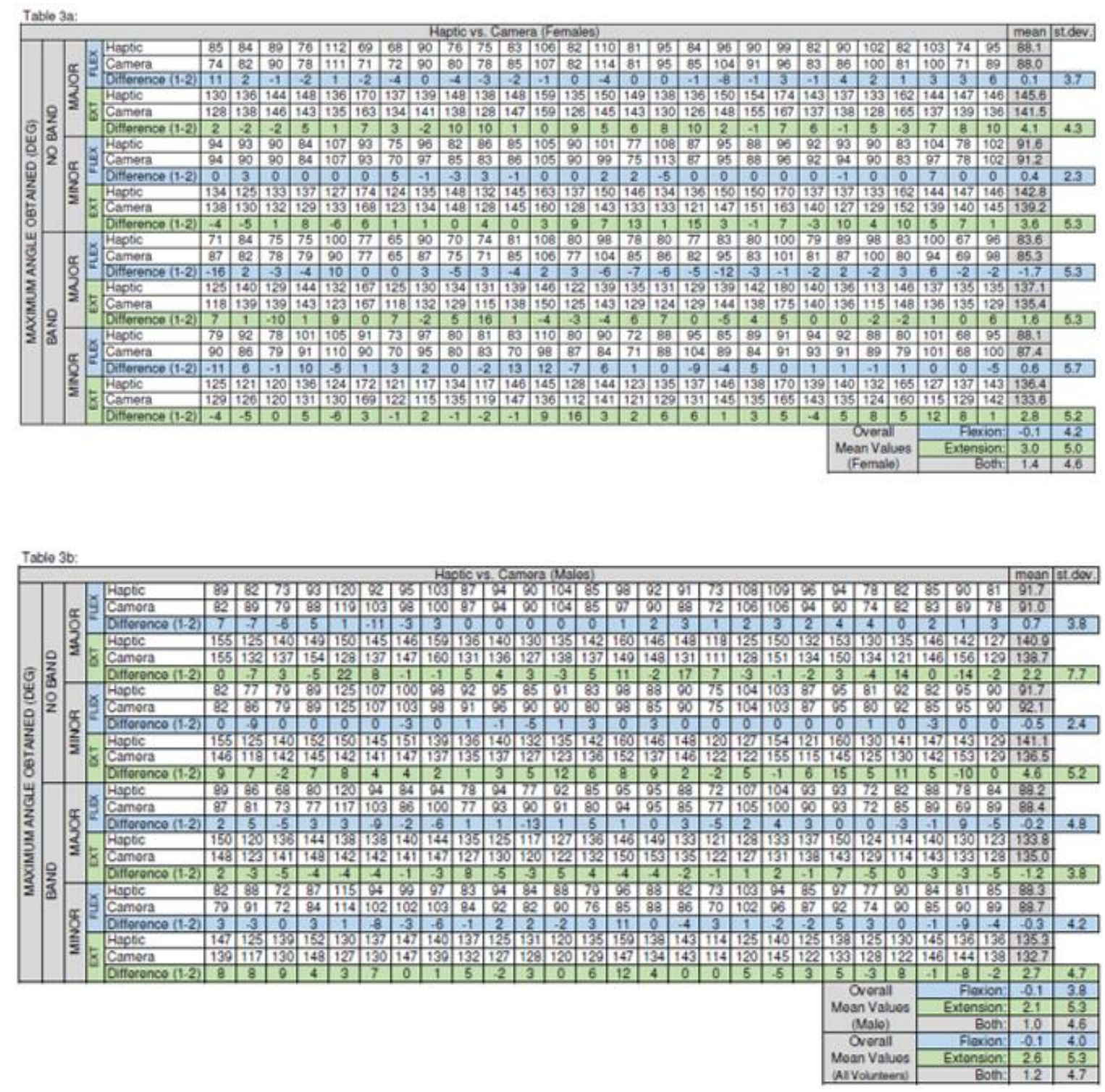

Table 3: Angle measurements from volunteer study illustrating haptic (in-person) data compared to camera (telemedicine) data.

\section{RESULTS}

The results of the first experiment, with three student researchers using three different smartphones to measure nine different known angles, are shown in Table 1. Across the three student researchers and the three smartphones, the average difference between measurements was $0.6^{\circ}$ and the average standard deviation was $0.3^{\circ}$ (All values have been rounded to the nearest tenth).

The results of the second experiment, where angles were drawn at random on a whiteboard and measured using both a protractor and the RateFast goniometer, are shown in Table 2.

In Table 2, the mean values in columns $4 \& 5\left(-1.1^{\circ}\right.$ and $0.7^{\circ}$, respectively) show that the angles measured with the RateFast goniometer are less than those measured with a protractor, averaging to a difference of $0.9^{\circ}$. The mean value in column 6 demonstrates that angle measurements by two separate individuals using the RateFast Goniometer app are $0.4^{\circ}$ on average. The standard deviations average to $1.5^{\circ}$. 
The results for the measurements of the maximum angles obtained in the third experiment are shown in Table 3, with results for females shown in Table 3A and results for males shown in Table 3B. As mentioned previously, the data was obtained from the study [5] in which the 53 volunteers (27 females and 26 males) checked their range of motion both with and without using a TheraBand; hence, Table 3 divides results into "band" and "no band" categories. The categories "major" and "minor" refer the volunteer using the "dominant" or "non-dominant" arm, respectively. Finally, both flexion and extension values were measured, as illustrated previously in Figures 1 and Figure 2. No significant difference is seen when comparing the data for males vs. females, major vs. minor arms, or Theraband vs. no Theraband.

Table 3 shows the range of motion data for the 53 volunteers, as well as the mean for each measurement category. The haptic mode measurements and the camera mode measurements have an average difference of $1.2^{\circ}$ and the standard deviation of the difference between haptic and camera measurements was determined to be $4.7^{\circ}$ across all volunteers.

\section{DISUCSSION}

When testing the accuracy of the measurements obtained by the RateFast goniometer app, a practical definition of "accuracy" must be agreed upon. Given the widespread use and practical application of the AMA Guides, we have adopted the functional definition of "accuracy" found in this text. As mentioned, when evaluating the accuracy of a set of measurements, the AMA Guides tolerates deviation between two measurements of up to $10 \%$ [3]. Within the context of the shoulder flexion plane, we consider $180^{\circ}$ to be $100 \%$ range of motion; in other words, an individual with a healthy shoulder can be expected to move their shoulder along the flexion plane to $180^{\circ}$. For the shoulder's extension plane, $40^{\circ}$ is considered $100 \%$ range of motion. Therefore, to be considered "accurate," two flexion measurements must be within $18^{\circ}$ of each other $\left(10 \%\right.$ of $180^{\circ}$ is $\left.18^{\circ}\right)$ while two extension measurements must be within $4^{\circ}$ of each other $\left(10 \%\right.$ of $40^{\circ}$ is $\left.4^{\circ}\right)$.

When applying the AMA Guides' standard of accuracy to the first experiment of this study, we find that the measurements obtained by the RateFast Goniometer app are accurate; the results fall within the error tolerance of $10 \%$.

In the results from the first experiment (Table 1) the average difference between measurements was $0.6^{\circ}$ and the average standard deviation was $0.3^{\circ}$, which are well beneath the error-tolerance of $18^{\circ}$ for flexion or $4^{\circ}$ for extension defined by the AMA Guides.

Similarly, in the second experiment (Table 2, columns 4, 5 , and 6) the mean values (under $1^{\circ}$ ) and the standard deviation values (averaging to $1.5^{\circ}$ ) of the results are within the acceptable range of accuracy defined by the AMA Guides for both flexion and extension shoulder planes.

In the third experiment, the in-person measurements obtained using the haptic mode of the RateFast goniometer app can be seen to deviate slightly in most cases, but on average are consistent with the camera measurements obtained using the RateFast goniometer app on a computer screen while viewing the pictures taken while the measurements were made. The haptic and camera measurements are, on average, within $1.4^{\circ}$ of each other, which is well within $18^{\circ}$ for the flexion plane and $4^{\circ}$ for the extension plane.

From our results, we can conclude that the RateFast goniometer app can be used to accurately measure angles according to the standards of accuracy set forth in the AMA Guides. Given the minor deviation in results between the haptic mode and the camera mode of the RateFast goniometer app, this digital goniometer application may be used to obtain accurate measurements 
both in telemedicine settings as well as in a doctor's office.

\section{ACKNOWLEDGEMENT}

We thank Sarah Alchemy (University of Puget Sound) for assistance in the determination of the calibration of the video goniometer for the study; we thank Chris Young PhD (Alverno College) for assistance in revising the paper; we thank the Honorable Steven Siemers for reviewing the discussion of the medical-legal implications of the study's results; we thank the 53 volunteers from the Hamline University student body. This research was funded by the Hamline University Kent H. Bracewell Scholarship Fund and the Barbara Lund Fund.

\section{REFERENCES}

1. Christenson J (2019) Handbook of biomechatronics. London: Academic Press.

2. Jones A, Sealey R, Crowe M, et al. (2014) Concurrent validity and reliability of the simple goniometer iPhone app compared with the universal goniometer. Physiotherapy Theory and Practice 30(7): 512-516.

3. Andersson G, Cocchiarella L (2006) AMA guides to the evaluation of permanent impairment. $5^{\text {th }}$ (Edn.) American Medical Association Press, USA.

4. Arun C (2015) RateFast Goniometer. RateFast Blog.

5. Johnson M (2020) Developing a quantitative approach to strength testing in telemedicine.

6. Zhao JZ, Blazar PE, Mora AN, et al. (2019) Range of motion measurements of the fingers via smartphone photography. HAND: 1558944718820955.

7. Ramkumar PN, Haeberle HS, Navarro SM, et al. (2018) Mobile technology and telemedicine for shoulder range of motion: Validation of a motion-based machine-learning software development kit. Journal of Shoulder and Elbow Surgery 27(7): 1198-1204.

8. Chanlalit C, Kongmalai P (2012) Validation of the telemedicine-based goniometry for measuring elbow range of motion. Journal of the Medical Ass ociation of Thailand 95(Suppl. 12): S113-S117.

9. Tachakra S, Lynch M, Newson R, et al. (2000) A comparis on of telemedicine with face-to-face consultations for trauma management. Journal of Telemedicine and Telecare 6(1_suppl): 178-181.

10. Smith AC, Kimble R, Mill J, et al. (2004) Diagnostic accuracy of and patient satisfaction with telemedicine for the follow-up of paediatric burns patients. Journal of Telemedicine and Telecare 10(4): 193-198.

11. Benger JR, Noble SM, Coast J, et al. (2004) The safety and effectiveness of minor injuries telemedicine. Emerg ency Medicine Journal 21(4): 438-445.

12. Saleh M, Schoenlaub S, Desprez P, et al. (2009) Use of digital camera imaging of eye fundus for telemedicine in children suspected of abusive head injury. British Journal of Ophthalmology 93(4): 424-428.

13. Hailey D, Roine R, Ohinmaa A (2002) Systematic review of evidence for the benefits of telemedicine. Journal of Telemedicine and Telecare 8(1_suppl): 1-7.

14. Hjelm NM (2005) Benefits and drawbacks of telemedicine. Journal of Telemedicine and Telecare 11(2): 60-70.

15. Abdoh AA, Krousel-Wood MA, Re RN (2003) Accuracy of telemedicine in detecting uncontrolled hypertension and its impact on patient management. Telemedicine Journal and e-Health 9(4): 315-323.

16. Oakley AM, Astwood DR, Loane M, et al. (1997) Diagnostic accuracy of teledermatology: Results of a preliminary study in New Zealand. The New Zealand Medical Journal 110(1038): 51. 
http://www.tridhas cholars .org | October-2020

17. Santamore WP, Homko CJ, Kashem A, et al. (2008) Accuracy of blood pressure measurements transmitted through a telemedicine system in underserved populations. Telemedicine and e-Health 14(4): 333-338.

18. Ramkumar P, Haeberle H, Navarro S, et al. (2018) Mobile technology and telemedicine for shoulder range of motion: validation of a motion-based machine-learning software development kit. Journal of Shoulder and Elbow Surgery 27(7): 1198-1204.

19. Alawna M, Unver B, Yuksel E (2019) The reliability of a smartphone goniometer application compared with a traditional goniometer for measuring ankle joint range of motion. Journal of the American Podiatric Medical Association 109(1): 22-29.

20. Lau C, Churchill R, Kim J, et al. (2002) Asynchronous web-based patient-centered home telemedicine system. IEEE Transactions on Biomedical Engineering 49(12): 1452-1462.

21. Steele L, Lade H, McKenzie S, et al. (2012) Assessment and diagnosis of musculoskeletal shoulder dis orders over the internet. International Journal of Telemedicine and Applications 2012: 945745.

22. Eriksson L, Lindström B, Gard G, et al. (2009) Physiotherapy at a distance: A controlled study of rehabilitation at home after a shoulder joint operation. Journal of Telemedicine and Telecare 15(5): 215-220.

23. Dreyer N, Dreyer K, Shaw D, et al. (2001) Efficacy of telemedicine in occupational therapy: A pilot study. Journal of Allied Health 30(1): 39-42.

24. Sandström J, Swanepoel D, Laurent C, et al. (2020) Accuracy and reliability of smartphone self-test audiometry in community clinics in low income settings: A comparative study. Annals of Otology, Rhinology \& Laryngology 129(6): 578-584.

25. Wibbenmeyer L, Kluesner K, Wu H, et al. (2016) Video-enhanced telemedicine improves the care of acutely injured burn patients in a rural state. Journal of Burn Care \& Research 37(6): e531-e538.

26. Nesbitt T, Hilty DM, Kuenneth CA, et al. (2000) Development of a telemedicine program. Western Journal of Medicine 173(3): 169-a-174. 